\title{
Device Incorrectly Cleaned During Reprocessing
}

National Cancer Institute

\section{Source}

National Cancer Institute. Device Incorrectly Cleaned During Reprocessing. NCI

Thesaurus. Code C139486.

The cleaning procedure is not followed correctly or used inappropriate cleaning materials. 